\title{
APPLICATION OF EXTENDED KALMAN FILTER IN PERSISTANT SCATTERER INTERFEROMETRY TO ENHACE THE ACCURACY OF UNWRAPPING PROCESS
}

\author{
A. Tavakkoli Estahbanati ${ }^{\text {a }}$, M. Dehghani ${ }^{\text {b }}$ \\ ${ }^{a}$ M.Sc. student of remote sensing of the Dept. of Civil and Environmental Engineering, School of Engineering, Shiraz University - \\ Amintavakkoli.est@gmail.com \\ ${ }^{\mathrm{b}}$ Assistant professor of the Dept. of Civil and Environmental Engineering, School of Engineering, Shiraz University, Iran - \\ Dehghani_rsgsi@yahoo.com
}

KEY WORDS: InSAR, Phase Unwrapping, Persistent Scatterer, irregular space, Extended Kalman Filter, Cost-map

\begin{abstract}
:
In interferometry technique, phases have been modulated between $0-2 \pi$. Finding the number of integer phases missed when they were wrapped is the main goal of unwrapping algorithms. Although the density of points in conventional interferometry is high, this is not effective in some cases such as large temporal baselines or noisy interferograms. Due to existing noisy pixels, not only it does not improve results, but also it leads to some unwrapping errors during interferogram unwrapping. In PS technique, because of the sparse PS pixels, scientists are confronted with a problem to unwrap phases. Due to the irregular data separation, conventional methods are sterile. Unwrapping techniques are divided in to path-independent and path-dependent in the case of unwrapping paths. A regiongrowing method which is a path-dependent technique has been used to unwrap PS data. In this paper an idea of EKF has been generalized on PS data. This algorithm is applied to consider the nonlinearity of PS unwrapping problem as well as conventional unwrapping problem. A pulse-pair method enhanced with singular value decomposition (SVD) has been used to estimate spectral shift from interferometric power spectral density in $7 * 7$ local windows. Furthermore, a hybrid cost-map is used to manage the unwrapping path. This algorithm has been implemented on simulated PS data. To form a sparse dataset, A few points from regular grid are randomly selected and the RMSE of results and true unambiguous phases in presented to validate presented approach. The results of this algorithm and true unwrapped phases were completely identical.
\end{abstract}

\section{INTRUDUCTION}

SAR Interferometry (InSAR) technique can be used for topography extraction and estimation ground surface displacements. Phase unwrapping is one of the most important parts displacements estimation from an interferogram (Chen \& Zebker, 2000). Phase unwrapping is a demanding process to reconstruct unambiguity from phases modulated between $0-2 \pi$. Most of conventional unwrapping InSAR methods unwrap phases based on nyquist-rate which is defined over a regular and continues space. Nevertheless, these methods are not effective in some cases such as large temporal baseline or vegetated areas which decorrelate interferograms (Dehghani, et al., 2013). PS InSAR methods are presented to overcome these drawbacks. Outputs of PSInSAR techniques lead to an irregular and discontinues space where conventional unwrapping methods are not applicable. Reducing an irregular space into a regular space is an approach to unwrap Persistent Satterer (PS) phases (Agram $\&$ Zebker, 2009). In order to reduce data into a regular grid, at first, interferogram is divided into some convex patches by means of Voronoi diagrams. Then, phase in each patch are interpolated by nearest neighbor methods. Thanks to the reduction into a regular and continues space all kind of conventional unwrapping methods can be implemented on PSInSAR results.

Conventional unwrapping algorithms based on how they define and model unwrapping problem can be divided into two methods (Chen \& Zebker, 2001). Some methods which try to solve the problem globally is called path-independent such as Lp norms (Ghiglia \& Romero, 1994). As these methods try to find a global solution, their results are not reliable in noisy areas. But if the power of norms is decreased, the accuracy of results will be increased (Chen \& Zebker, 2000).

In spite of global unwrapping methods, if the power of norms is considered as zero, problem reduces to minimize the weighted number of locations where the unwrapped and the wrapped gradients differ (Chen \& Zebker, 2000). Path following methods expressly identify and accommodate discontinuities during direct integration of phase gradients. Therefore, path following unwrapping algorithms follow a path in the wrapped phase and unwrap each pixel locally. There are a vast majority of various path following unwrapping methods which utilize a local unwrapping function and unwrap all the pixels one-by-one. Thus, the process of local unwrapping can be divided in to two main step. The path which is used to guide the unwrapping process and the unwrapping function. Sequential paths such as branch-cut (Goldstine, et al., 1988) and MST (Chen \& Zebker, 2000) are the simplest paths used to manage the direction of unwrapping. The main goal of sequential paths is to locate and avoid discontinuities and any type of area filling curves such as flood fill can be used to unwrap phases. These paths are generally combined with filter based unwrapping functions (Osmanoglu, et al., 2011; Chirico \& Schirinzi, 2012). Unlike sequential paths, region growing algorithms such as Phase derivative variance (PDV) (Osmanoglu, et al., 2011), Maximum Coherency (MC) (Ghiglia \& Pritt, 1998), Least Squares (LS) (Ghiglia \& Romero, et al., 1994) use cost or quality maps to define preferred unwrapping path. Cost maps as well as quality maps can help algorithms to unwrap phases with higher qualities in advance.

Because of sparse distribution of PSs, lots of phase information has been lost. Thus, unwrapping in a discontinuous space is more demanding and it is essential that phases be unwrapped precisely 
(Ferretti, et al., 2001). Therefore, the main goal of this paper is to increase the accuracy of unwrapping phases in irregular space by considering Extended Kalman Filter (EKF) as unwrapping function. Presented approach has been implemented on simulated data where the accuracy of proposed approach is compared with the results of PDV in different range of densities.

This paper is organized as follows. Section 2 explains steps to unwrap sparse phases with presented approach. Unwrapping results and validation are presented in Section 3. Concluding remarks are presented in section 4.

\section{METHODOLOGY}

\section{2-1- Interpolate phases into a regular grid}

Finding the number of integer cycles which is missed in inreferomtric phases is the main goal of unwrapping algorithms. As conventional unwrapping algorithms can be used in a regular and continues 2-D space, sparse phases should be interpolated into a regular grid such that the phase residues be conserved in the interpolated 2-D regular grid. It can be shown that nearest neighbor interpolation methods can maintain these assumption (Agram \& Zebker, 2009). At first, all PS pixels will be connected to each other with Delaunay triangles where PSs are the vertex of triangles, then Voronoi polygons are used to divide an interferogram into some convex polygons which need to be interpolated by nearest neighbor methods. After that phases are reduced in to a regular grid all types of conventional unwrapping methods can be used to unwrap phases.

\section{2-2- Nonlinear phase unwrapping}

It should be noticed that because of sparse distribution of data, phases need to be unwrapped precisely. In this paper, Kalman Filter is implemented on interpolated phases. It is extremely noticeable that phase unwrapping is a nonlinear problem, thus nonlinear solutions such as Extended Klaman Filters can achieve a better results (Osmanoglu, et al., 2009; Guo-lin, et al., 2010). There are various methods which can be used to estimate phase slope (Martinez-Espla \& Martinez-Marin, 2009; Liu, et al., 2010). In this work, SVD algorithm is used in prediction step to increase the accuracy of phase slope estimation (Xie \& Li, 2014). In this section, some general information about Kalman Filter to unwrap phases are presented here. Kalman Filter $(\mathrm{KF})$ is a recursive filter considering observational model for relating different observations to a combined state ( (Kalman, 1960)). In the case of unwrapping problem, this observational model would define a model required to link interferometric wrap phases to unambiguous phases. Therefore, complex interferometric phases can be convert to unwrapped phases as follows:

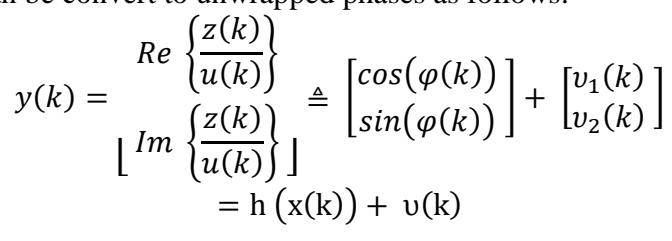

Where $\mathrm{y}(\mathrm{k})$ is the real and complex parts of interferometric observations, $\varphi(k)$ is unambiguous phases. In conventional unwrapping the noise processes $\mathrm{v} 1(\mathrm{k})$ and $\mathrm{v} 2(\mathrm{k})$ are considered as a zero-mean white Gaussian (even though the Gaussianess assumption is not correct in the strict sense, practically not very crucial); however, since PS pixels have persistent phase characteristic during time, the noise process are assumed to be zero.

Equation 1 explains a simple but extremely effective state-space model presented in (Loffeld, et al., 2008) to unwrap phases.

$$
\mathrm{x}(\mathrm{k}+1)=\mathrm{A} \cdot \mathrm{x}(\mathrm{k})+\hat{\mathrm{u}}(\mathrm{k})+\omega(\mathrm{k})
$$

Where $\mathrm{x}(\mathrm{k})$ is an the unwrap phase of current pixel, $\mathrm{A}$ is the transition matrix which is considered as identity matrix, $\hat{\mathrm{u}}(\mathrm{k})$ is the gradient of unwrap phases between current pixel and the next one and $w(k)$ is the uncertainty of phase slope estimation which can be seen as noise covariance matrix . In prediction step, each pixel is unwrapped based on the phase slope estimated from its wrapped neighbors. It can be pointed out that phase slope between two pixels is related to the mean fringe frequency which can be estimated from power spectral density in a local window $\left(m^{*} n\right)$. It is essential that phase slope be estimated precisely, so Pulse Pair method (Frehlich \& Yadlowsky, 1994) which is enhanced by SVD is used to estimate the mean fringe frequency in 2-D interpolated space. Thus, the noise covariance matrix can be obtain as follows:

$$
q_{\Delta \varphi}=(2 \pi)^{2}\left[(m-a)^{2} c\left(f_{x}\right)+(\mathrm{n}-\mathrm{s})^{2} c\left(f_{y}\right)\right]
$$

Where $c\left(f_{x}\right)$ and $c\left(f_{y}\right)$ are the mean frequency estimated in range and azimuth direction, respectively. $(a, s)$ is the position of current pixel and $(m, n)$ is the position of next pixel which must be unwrapped.

The correction step compares the interferometric observation with predicted value. Then, values of predicted state and error covariance will be corrected with new values which are calculated with current observations. So the correction step can be summarized as:

$$
\begin{gathered}
\hat{x}_{k+1}=\hat{x}_{k}^{+}+\hat{u}_{k} \\
P_{k+1}^{-}=P_{k}^{+}+q_{k} \\
\left.K(k+1)=P_{k+1}^{-}(k+1) \cdot C_{F}^{T}(k+1) \cdot\right]^{-1} \\
{\left[C_{F}(k+1) \cdot P^{-}(k+1) \cdot C_{F}^{T}(k+1)+R(k+1)\right]} \\
\hat{\mathrm{x}}_{\mathrm{k}+1}^{+}=\hat{\mathrm{x}}_{\mathrm{k}+1}^{-}+\mathrm{K}(\mathrm{k}+1) \cdot \mathrm{r}_{\mathrm{k}+1} \\
\mathrm{P}^{+}(\mathrm{k}+1)=\mathrm{P}^{-}(\mathrm{k}+1)-\mathrm{K}(\mathrm{k}+1) \\
\cdot \mathrm{C}_{\mathrm{F}}(\mathrm{k}+1) \cdot \mathrm{P}^{-}(\mathrm{k}+1)
\end{gathered}
$$

Where $P_{k+1}^{-}$is predicted covariance matrix, $K(k+1)$ is filter gain value calculated as correction weights from previous steps up to now, $\mathrm{r}_{\mathrm{k}+1}$ is correction term,$P_{k+1}^{+}$is the covariance matrix of correction step, and $\mathrm{C}_{\mathrm{F}}(\mathrm{k}+1)$ is the first derivation of observation matrix.

$$
\begin{aligned}
C_{\mathrm{F}}(\mathrm{k}+1)=\frac{\mathrm{d}}{\mathrm{dx}} \mathrm{h}[\mathrm{x}]_{\mid \hat{\mathrm{x}}_{\mathrm{k}+1}^{-}} \\
\quad=\left[-\sin \left(\hat{\mathrm{x}}_{\mathrm{k}+1}^{-}\right) \cdot \cos \left(\hat{\mathrm{x}}_{\mathrm{k}+1}^{-}\right)\right]^{\mathrm{T}}
\end{aligned}
$$

where $\frac{\mathrm{d}}{\mathrm{dx}}$ is the derivation of observation model.

\section{2-3- Cost map}

As it mentioned local unwrapping methods such as KF use costmaps to control unwrapping path. Several methods such as PDV, MC, PDV_Branch cut are presented to introduce cost or quality maps. Due to the sparse distribution of phases and reducing data into a regular space, unwrapping process should be managed critically .Therefore, a PDV cost map enhanced by geometrical information and the distribution of PS is used to increase the accuracy of unwrapping presses.

\section{3- RESULTS AND DISCUSSION}

\section{3-1- Data set}


In this section, presented approach is applied to a simulated data set. True unwrap phases containing a high deformation rate are wrapped to make a wrap interferogram where some pixels are selected randomly to build a sparse and irregular space. The efficiency and robustness of proposed approach has been investigated in different density of PSs. Likewise the definition of PS which means that they have high phase stability (Hooper, et al., 2004), the simulated interferogram is considered without noise. In order to demonstrate the advantages of our method, the results of PDV is compared with proposed approach. As some other cost maps such as coherency can not be defined on sparse data unlike conventional unwrapping, PDV cost map because of considering the information of phases is used to control the unwrapping path.

\section{3-2- Validation}

In validation step, the Root Mean Square Error (RMSE) between proposed approach and true unambiguous phases is compared to the result of PDV unwrapping method. It is important to know how the distribution and density of PS can diminish the result of unwrapping. Thus the RMSE of unwrapped phase in different densities is considered to confirm the merits of propose approach. Figure 1.a shows the simulated true unwrap phases. Moreover, the Wrap interferogram which is made by modulation of true unambiguous phases is demonstrated in figure 1.b.

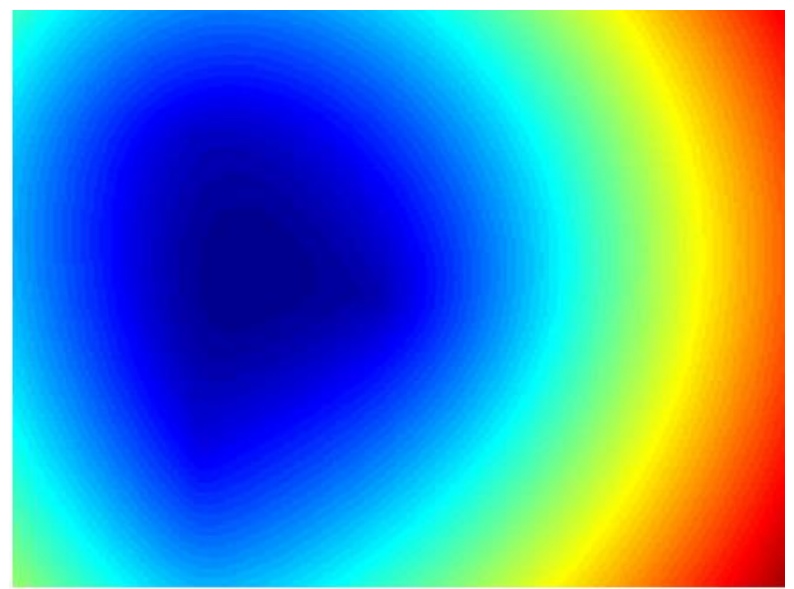

a)

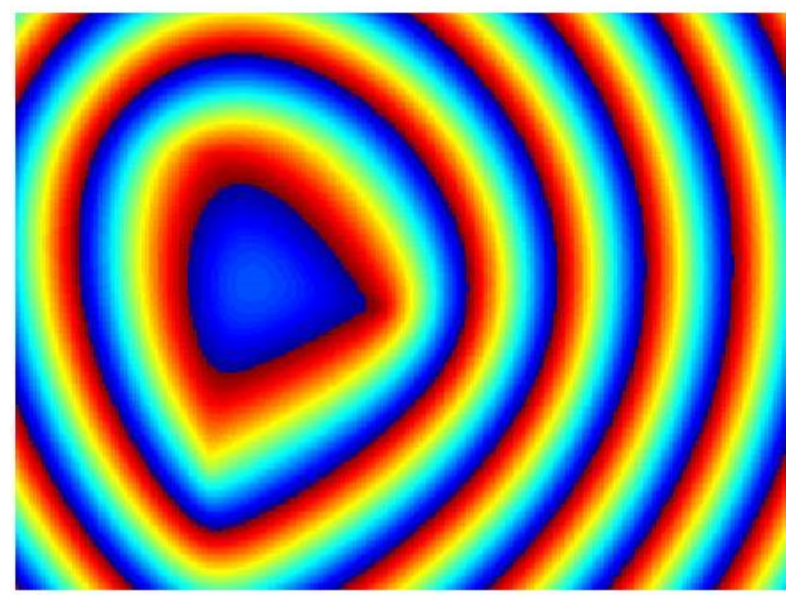

b)

Figure 1. Simulated phases. a) True unambiguous interferogram. b) An interferogram of wrap phases
An irregular space which is constructed by random selection of figure1.b pixels and the 2_D continuous interferogram interpolated with nearest neighbor method are presented in figure 2.a and 2.b, respectively.

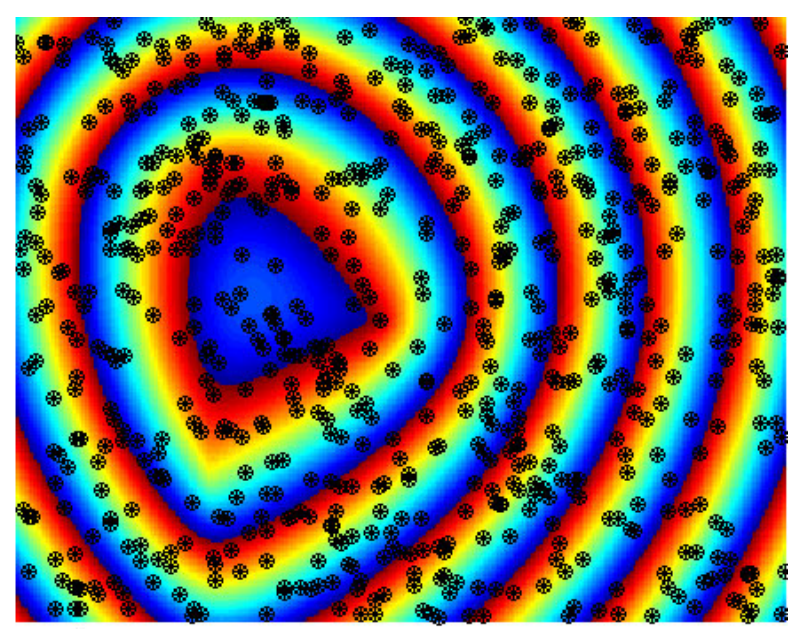

a)

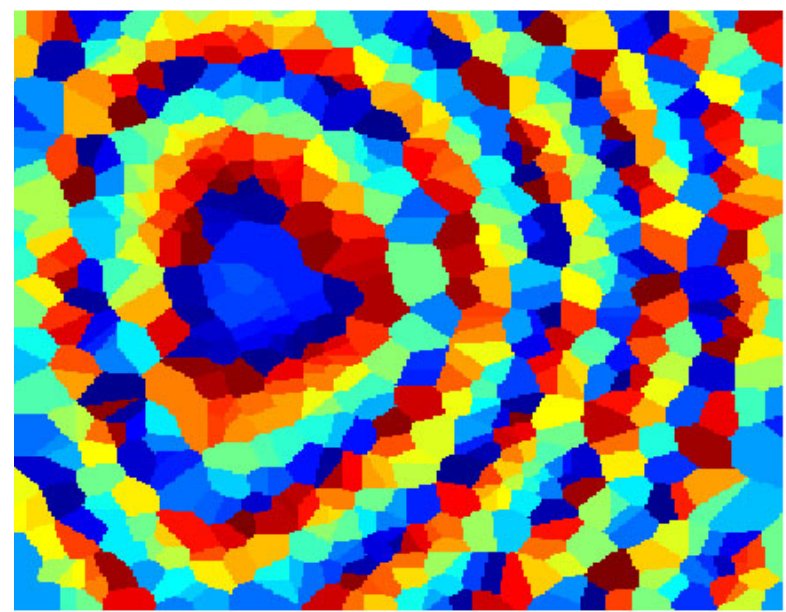

b)

Figure 2. A simulated sparse interferogram. a) An example of pixels selected as PSs. Black point depict the location of PSs. b) A 2-D interpolated interferogram of wrap phases.

After building the hybrid cost map, EKF unwrapping is implemented on interpolated phases. In order to shows the ability of presented approach to increase the accuracy of unwrapping process, interferograms which is unwrapped by presented approach and PDV method are revealed in figure3. In order to make an irregular space paces only 1 percent of pixels are selected as PS. 


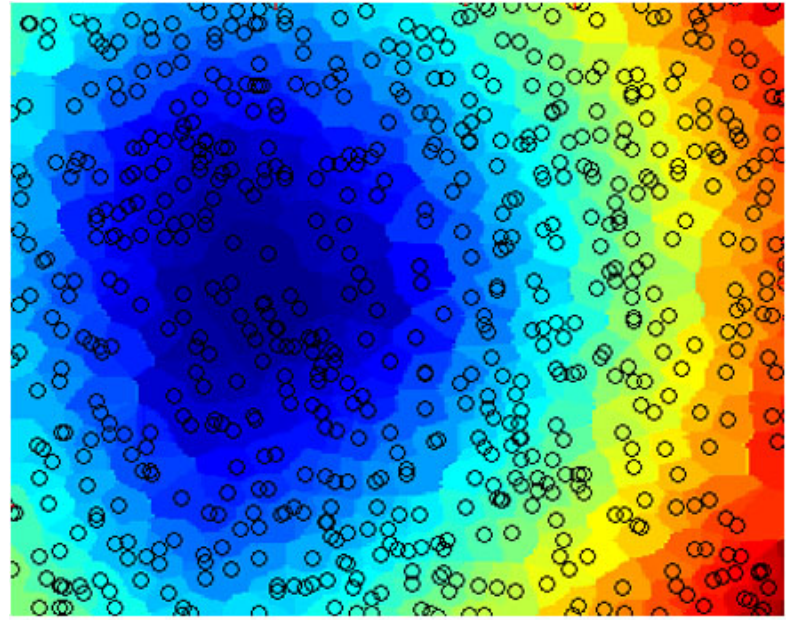

a)

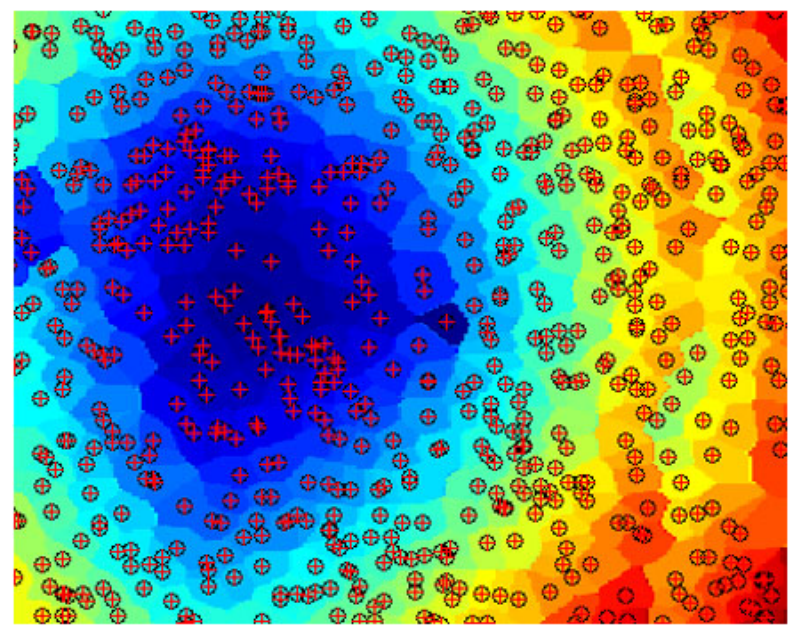

b)

Figure 3. Unwrapped interferograms. a) An unwrapped interferogram which is the result of proposed approach. $b$ )

An interferogram is unwrapped by PDV method. Black circles shows PS which is unwrapped correctly, while circles filled by red crosses are unwrapped incorrectly.

It is noticeable that presented approach has unwrapped phases correctly, while PDV was completely unable to unwrap phases accurately. The RMSE of true unwrap phases and the result of presented approach is depicted in figure 4 .
A considerable drop can be seen in graph where the density of pixels is about $0.6638 \%$. However, PDV method is sterile in larger densities such as $1.67 \%$. Moreover, some fluctuations which is the effect of distribution of PSs can be seen. Therefore, it can convey that considering the distribution of PSs can be effective to enhance the results of unwrapping phases

\section{4- CONCLUSION}

. As unwrapping is a key step to estimate deformation from an interferogram either in conventional or PS techniques, several methods have been proposed to unwrap PS data. Reducing phase into a regular grid can present an effective solution for unwrapping sparse phases. Thanks to the reduction into a regular space all kind of unwrapping methods can be used to unwrap sparse phases. Path-dependent techniques can be divided into sequential path and region-growing. Using Delaunay triangulation to reduce sparse data on a regular grid makes strict polygon boundary, hence moving from one polygon to other neighbor polygons is too rough; however, edges can be passed gradually and smoothly by means of Kalman Filter.

Moreover, a cost-map is defined to prioritize PSs which should be unwrapped. The cost-map consists of PDV which is enhanced with geometric properties and neighbor polygon distributions. This algorithm has been implemented on simulated PS data. To form a sparse dataset, $1.67 \%$ of points from regular grid are selected randomly and the sparse wrap interferogram unwrapped by proposed method precisely, while the PDV was not able to unwrap phases correctly. In addition, the accuracy of proposed approach in different densities assessed which reveals that the proposed approach is applicable in lower densities.

\section{REFRENSES}

Agram, P. S., \& Zebker, H. A. (2009). Sparse Two-Dimensional Phase Unwrapping Using Regular-Grid Methods. IEEE Geoscience and Remote Sensing Letters, 6(2), 327-331.

Chen, C. W., \& Zebker, H. A. (2000). Network apploaches to two-dimentional phase unwrapping intractability and two new algorithm. The Optical Society of America A, 18(3), 401-414.

Chen, C. W., \& Zebker, H. A. (2001). Two-dimentional phase unwrapping with use of statistical models for cost function in nonlinear optimization. The Optical Society America A, 18(2), 338-351.

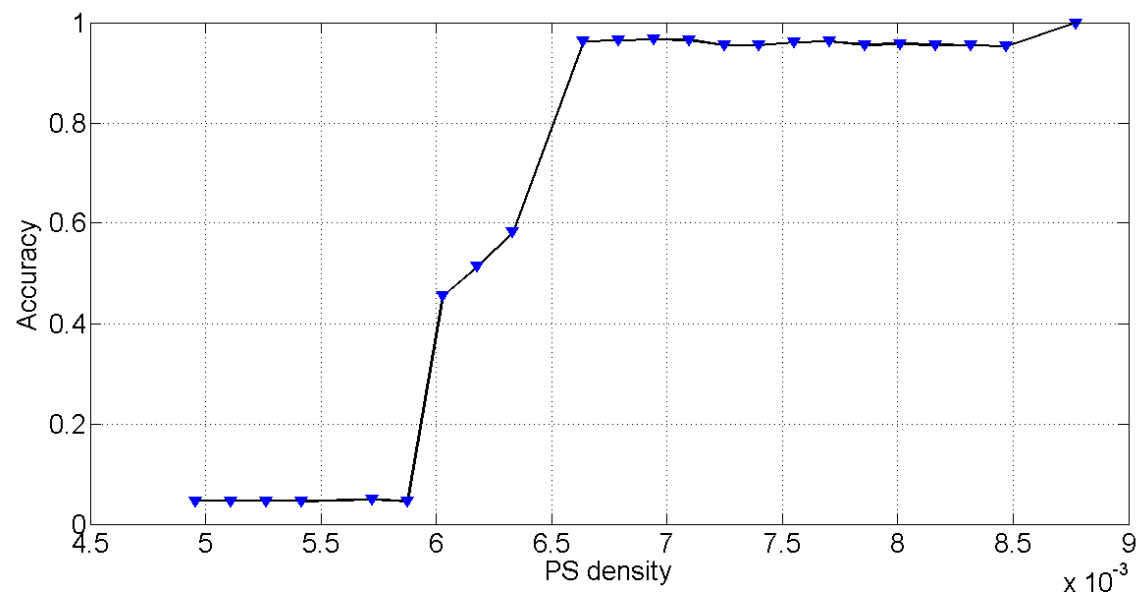

Figure 4. Accuracy of proposed approach against different PS densities. 
Chirico, D., \& Schirinzi, G. (2012). INSAR PHASE UNWRAPPING USING NONLINEAR K ALMAN SMOOTHER. Geoscience and Remote Sensing Symposium (IGARSS), 2012 IEEE International (pp. 5610-5613). Munich, Germany : IEEE .

Dehghani, M., Valadan Zoej, M. J., Hooper, A., Hanssen, R. F., Entezam, I., \& Saatchi, S. (2013). Hybrid Conventional and Persistent Scatterer SAR Interferometry for land subsidence monitoring in the Tehran Basin, Iran. ISPRS Journal of Photogrammetry and Remote Sensing, 79, 157-170.

Ferretti, A., Prati, C., \& Rocca, F. (2001). Permanent Scatterers in SAR Interferometry. IEEE Transactions on Geoscience and Remote Sensing, 39(1), 8-20.

Frehlich, R. G., \& Yadlowsky, M. J. (1994). Performance od Mean-Frequency Estimation for Doppler Radar and Lidar. American Meteorogical Society, 41, 1217.

Ghiglia, D. C., \& Pritt, M. D. (1998). Two-Dimentional Phase Unwrapping: Theory, Algorithms and Software. WileyBlackwell.

Ghiglia, D. C., \& Romero, L. A. (1994). Robust two-dimensional weighted and unweighted phase unwrapping that uses fast transforms and iterative methods. The Optical Society of America A, 11(1), 107-117.

Goldstine, R. M., Zebker, H. A., \& Werner, C. L. (1988). Sattelite radar Interferometry : Two-dimentional phase unwrapping. Radio Science, 23(4), 713-720.

Guo-lin, L., Hua-dong, H., \& Qiu-xiang, T. (2010). The Implementation and Analysis of Kalman Filter Phase Unwrapping Algorithm of Insar

Hooper, A., Zebker, H. A., Paul, S., \& Kampes, B. (2004). A new method for measuring deformation on volcanoes and other natural terrains using InSAR persistent scatterers. Geophysical Research Letters, 31(23).

Kalman, R. E. (1960). A New Approach to Linear Filtering and Prediction Problems. Transactions of the ASMEJournal of Basic Engineering, 35-45.

Liu, G., Hao, H., Yang, F., Yan, M., Du , Z., \& Dang, Y. (2010). InSAR Kalman Filter phase unwrapping algorithm based on topographic factors. OCEANS 2010 (pp. 1-6). Sydney, Sydney, NSW: IEEE.

Loffeld, O., Nies, H., Knedlik, S., \& Yu, W. (2008). Phase Unwrapping for SAR Interferometry-A Data Fusion Approach by Kalman Filtering. IEEE Transactions on Geoscience and Remote Sensing, 46(1), 47-58.

Martinez-Espla, J. J., \& Martinez-Marin, T. (2009). An optimized algorithm for InSAR phase unwrapping based on particle filtering, matrix pencil, and regiongrowing techniques. IEEE Geoscience and Remote Sensing Letters, 6(4), 835-839.

Osmanoglu, B., Dixon, T. H., Wdowinski, S., \& Cabral-Cano, E. (2011). On the importance of path for phase unwrapping in synthetic aperture radar interferometry. Applied Optics, 50(19), 3205-3220.

Osmanoglu, B., Wdowinski, S., Timothy , D. H., \& Biggs, J. (2009). InSAR phase unwrapping based on extended Kalman filtering. Radar Conference, 2009 IEEE. Pasadena, CA, USA: IEEE.

Xie, X., \& Li, Y. (2014). Enhanced phase unwrapping algorithm based on unscented Kalman filter, enhanced phase gradient estimator, and path-following strategy. Applied Optics, 53(18), 4049-4060.

Xie, X., \& Pi, Y. (2011). Phase noise filtering and phase unwrapping method based on unscented Kalman filter. Journal of Systems Engineering and Electronics, 22(3), 365-372. 